\title{
An Alternative Mechanism for the 1,4-Asymmetric Induction in the Stereoselective Addition of $(R)$-Pantolactone to 2-Phenylpropylketene
}

\author{
Alexander M. Silva, ${ }^{a}$ Clarissa O. da Silva, ${ }^{*, a}$ André G. H. Barbosa,${ }^{b}$ Rosane A. Fontes, ${ }^{a}$ \\ Sergio Pinheiro, ${ }^{b}$ Marco Edilson F. Lima ${ }^{a}$ and Rosane N. Castro ${ }^{a}$ \\ ${ }^{a}$ Departamento de Química, Universidade Federal Rural do Rio Janeiro, Rodovia BR 465, km 47, \\ 23890-000 Seropédica-RJ, Brazil
}

${ }^{b}$ Instituto de Química, Universidade Federal Fluminense, 20141-020 Niterói-RJ, Brazil

\begin{abstract}
Um mecanismo alternativo para a indução assimétrica 1,4 , na adição estereosseletiva da $(R)$-pantolactona ao 2-fenilpropilceteno foi investigado teoricamente. Um mecanismo envolvendo uma transferência de hidrogênio intermolecular foi proposto, que considera a participação ativa da dimetiletilamina e seu íon como agentes de transferência de hidrogênio. No primeiro passo, uma molécula de dimetiletilamina neutra interage com o anel de 7 membros do enol intermediário. Uma interação ácido-base específica é estabelecida entre o grupo hidroxila do enol e o átomo de nitrogênio da amina. A amina neutra é básica o suficiente para remover o hidrogênio. Uma outra molécula de amina protonada doa seu hidrogênio à ligação dupla $\mathrm{C}=\mathrm{C}$, para fornecer os produtos desejados. A estereoquímica foi definida pelo modo como as moléculas de amina neutra e protonada aproximam-se do enol. O excesso diasteroisomérico encontrado concorda com o dado experimental [para $(S, R)$ e $(R, R)$ é de 99:1].
\end{abstract}

An alternative mechanism for the 1,4-assymmetric induction in the stereoselective addition of $(R)$-pantolactone to 2-phenylpropylketene was theoretically investigated. A mechanism involving an intermolecular proton transfer assisted by two amine molecules was proposed, which considered the active participation of the dimethylethylamine and its ion as proton transfer agents. In the first step, a neutral dimethylethylamine interacts with the seven-membered ring of the enol intermediate. A specific acid-base interaction is established between the hydroxyl group of the enol and the nitrogen atom of the dimethylethylamine. The neutral dimethylethylamine is basic enough to remove the proton. Another protonated dimethylethylamine is considered to donate its proton to the $\mathrm{C}=\mathrm{C}$ double bond to give the desired products. The stereochemical outcome was defined by the way that the neutral and protonated dimethylethylamine approached to the enol. The diastereoisomeric ratio found is in good agreement with experiments [for $(S, R)$ and $(R, R)$ it is 99:1].

Keywords: density functional calculations, diastereoselectivity, protonation, alcohols, ketenes

\section{Introduction}

Diastereoselective addition of a chiral alcohol to a ketene was reported firstly in 1919. ${ }^{1}$ Since then, several groups have tried to improve the diastereoisomeric excess (\% d.e.) of the final product, being successful only those that use alcohols of limited availability as chiral reagents..$^{2-4}$ However, in 1989, Larsen et al..$^{5}$ at Merck developed a synthetic route where naturally occurring $\alpha$-hydroxy esters were used as chiral inductors, yielding very promising d.e.'s. They had reported that chiral 2-arylpropionic esters

*e-mail: clarissa-dq@ufrrj.br were obtained with a diastereoisomeric excess as high as 99\% via a reaction of arylmethylketenes generated in situ with a variety of optically active $\alpha$-hydroxy esters such as $(R)$-pantolactone. Since Larsen et al..$^{5}$ have published their results, stereoselective addition of a chiral alcohol to prochiral ketenes derived from 2-aryl propionic acid has been widely discussed in the literature. ${ }^{6-9}$

In this reaction the goal is to transform racemic $\alpha$-substituted carboxylic acids into their enantiomerically enriched esters derivatives. The ketene formation and the addition of chiral alcohol normally occur in a one-step procedure being followed by a stereoselective protonation of the ketene. Since these chiral esters can be hydrolyzed 
without significant racemization, this process is useful for preparing various types of chiral $\alpha$-substituted carboxylic acids with important anti-inflammatory properties (some examples are ibuprofen, naproxen, ketoprofen, flunoprofen, etc). ${ }^{10,11}$

Some studies have shown that the base catalyzed addition of the chiral alcohol to ketenes derived from various racemic aryl propionic acids can furnish only one diastereoisomer. ${ }^{5}$ Cannizzaro et al. ${ }^{6,9}$ recently explored this reaction using quantum mechanical calculations and provided a quantitative model for its mechanism that is the most accepted in the literature. They carried out a very detailed theoretical study of the stereoselective additions of chiral alcohols, considering different chiral inductors as $(S)$-methyl lactate, $(S)$-3-methyl-2-butanol, and $(S)$-pantolactone to ketenes. The proposed mechanism for the first two chiral inductors states that the alcohol addition to the ketene catalyzed by trimethylamine is the rate determinant step, while the keto-enol tautomerization is the stereoselectivity determinant step. For (S)-pantolactone instead, the rate and stereoselectivity determinant steps are the same, the alcohol addition, because the bond rotation that would furnish a racemic mixture is precluded by a $10.9 \mathrm{kcal} / \mathrm{mol}$ energy barrier (see Figure 23 of reference 6). However, in all cases the authors always propose a transition state with only one amine molecule catalyzing the intramolecular keto-enol tautomerization, in a conducted tour mechanism ${ }^{12,13}$ It should be noted that in the solvation model used, the calculation of the non-electrostatic contribution was absent.

In the present work the synthetic route ${ }^{14}$ for the preparation of 2-phenyl-propionate pantolactonyl ester is theoretically revisited. Although it is not the main focus of this work, the parallel experiment is here mentioned to justify some choices of the theoretical description. The synthesis of this compound is outlined in Scheme 1. The alkylation of ester 1 (methyl phenylacetate) was achieved in good yield giving $\mathbf{2}$ (methyl 2-phenylpropanoate) as previously described in the literature. ${ }^{15}$ Subsequent hydrolysis furnished rac-3 (2-phenylpropionic acid) ${ }^{16}$ which was quantitatively converted into 2-phenylpropionyl chloride 4. The dehydrochlorination of $\mathbf{4}$ by dimethylethylamine at room temperature produced the methylphenylketene 5 , which upon treatment with $(R)$-pantolactone led to the mixtures of the chiral esters $6(S, R)$ - and $(R, R)$ phenylpropionic acid, $\alpha$-methyl-,tetrahydro-4,4-dimethyl2-oxo-3-furanyl esters in a 99: 1 ratio $(98 \%$ d. e. $){ }^{14}$

Thus, ketene formation and addition of $(R)$-pantolactone, a very efficient commercially available chiral auxiliary, occurred in one step procedure. The asymmetric transformation of $\mathrm{rac}-\mathbf{3}$ involves the stereoselective addition of a chiral alcohol to the corresponding prochiral ketene $\mathbf{5}$ followed by deracemizing protonation of this intermediate. The tertiary base, used in excess, both catalyzed the diastereoselective addition of an alcohol to the ketene and increased the stereoselectivity.

Ketene intermediate (methylphenylketene $\mathbf{5}$ in Scheme 1) is generated in situ in the reaction between acyl chloride (4) and tertiary amines (as dimethylethylamine). Ketene reacts immediately with the $(R)$-pantolactone to give intermediate $\mathbf{7}(\mathbf{I})$, which is in equilibrium with the enol 7(II) through a hydrogen shift (Scheme 2).

To understand the deracemizing protonation of ketene in the stereoselective formation of $(R, S)$-6 by using $(R)$ pantolactone as chiral inductor, the theoretical route we have investigated starts from intermediates $\mathbf{7}(\mathbf{I})$ and $\mathbf{7}$ (II) (which occurs after the alcohol addition to the ketene, as proposed in reference 6) and takes two different paths. Both paths involve proton transfer reactions, and the mechanisms considered for them were: (A) a direct intramolecular proton transfer not assisted by any amine molecule and (B) an intermolecular two-step proton transfer catalyzed by both, an amine molecule which abstracts the proton and an ammonium salt (an efficient proton donor formed in the<smiles>CCOC(=O)C(C)c1ccc(-c2ccc(C(C)OC(=O)Cc3ccccc3CC(C)=O)cc2)cc1</smiles>

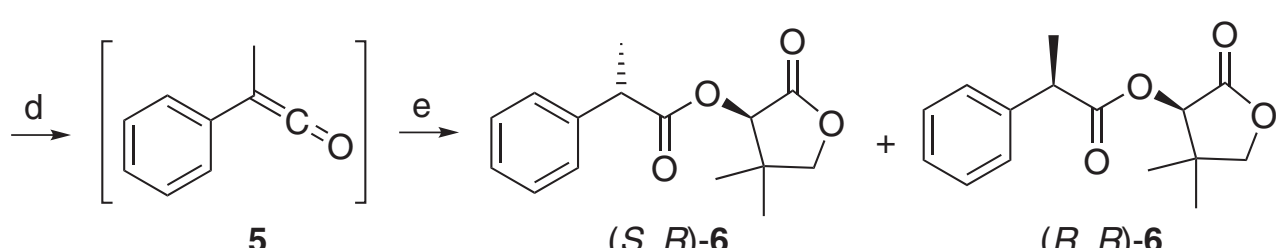

5

$(S, R)-6$

$(R, R)-6$

Scheme 1. (a) THF, $\mathrm{NaH}(0.1 \mathrm{~mol}), \mathrm{CH}_{3} \mathrm{I}(0.15 \mathrm{~mol})$, reflux, $3 \mathrm{~h}, 83 \%$; (b) $\mathrm{KOH} / \mathrm{EtOH}$, r.t., overnight, $90 \%$; (c) $\mathrm{SOCl}_{2}$, reflux, $3 \mathrm{~h}, 97 \%$; (d) $N, N$ dimethylethylamine ( 1.5 equiv.), $0{ }^{\circ} \mathrm{C}, 30 \mathrm{~min}$; (e) $(R)$-pantolactone (1.1 equiv., $0.2 \mathrm{~mol} \mathrm{~L}^{-1}$ in THF), $0{ }^{\circ} \mathrm{C}$ to r.t., $83 \%$, $98 \%$ d. e. 
<smiles>C#CC(=O)O[C@H]1C(=O)OCC1(C)C</smiles>

Scheme 2. Addition of $(R)$-pantolactone to ketene, generated in situ with simultaneous formation of an ammonium salt.

reaction medium when $\mathrm{HCl}$ is generated as a subproduct of the in situ ketene generation). This proposition is different from Cannizzaro's et al. ${ }^{6,9}$ proposition, in which the same amine molecule, through the conducted tour mechanism, assists the tautomerization ${ }^{12,13}$ (see Scheme 3). It should be noted that the tertiary amine we have used was dimethylethylamine, while that in reference 6 was trimethylamine.

\section{Methodology and Computational Details}

The calculations were performed at the DFT/B3LYP 17,18 level with the $6-311 \mathrm{G}(\mathrm{d}, \mathrm{p})$ basis set, using the Jaguar ${ }^{19}$ and Gaussian $03^{20}$ programs. Geometry optimization calculations were carried out to obtain the structures of reactants, products and transition states. After that, their vibrational frequencies were obtained in order to check whether the stationary points were correctly located. That is, if there were just real frequencies for reactants and products, and only one imaginary frequency for the transition state associated with the broken bond. The minimum energy path connecting these structures was obtained through an intrinsic reaction coordinate (IRC) calculation. ${ }^{21,22}$ There has been some concern in the literature about the possible inability of the B3LYP functional to accurately calculate reaction barrier heights in some systems containing valence electron-rich atoms. ${ }^{23}$ To verify if our results are to any extent compromised by these facts, we have recalculated the barrier's heights with the ab initio Moller-Plesset second

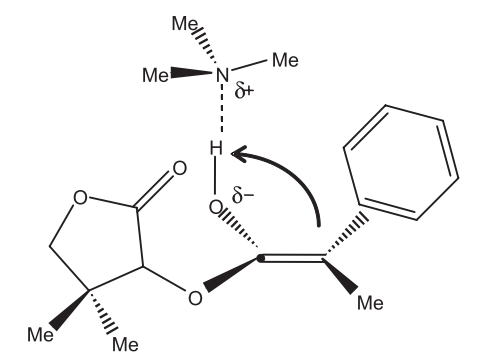

order (MP2) method. These calculations were performed with the same basis set, on the geometries found for reactants, intermediates and transition states species involved in both reaction paths, as single point energy calculations, just to check the relative energy values found from the density functional theory description.

The initial structures were obtained from GaussView ${ }^{24}$ All calculations were performed in gas-phase and in THF, since it is the solvent utilized in the experiments (see Scheme 1). Although this is an aprotic solvent, with low relative permittivity and then assumed to work only as a convenient dispersing media, the differential solvation effects should not be neglected. Therefore the system was also studied considering them, which were determined using the integral equation formalism (IEF) ${ }^{25}$ version of the polarized continuum solvation model (PCM). ${ }^{26,27}$ In this approach the solute is represented as a quantum mechanical charge distribution inside a cavity of molecular shape immersed in a macroscopic dielectric with known relative permittivity $\varepsilon$. The electrostatic interaction between solute and solvent can then be represented in terms of a set of apparent surface charges located on the cavity. Such cavity is built from interlocking spheres centered on atoms or group of atoms. The radii of these spheres are $1.80 \AA$ for $\mathrm{N}$ and $\mathrm{O}$ atoms, $2.28 \AA$ for $\mathrm{CH}$ or $\mathrm{CH}_{2}$ group of atoms, $2.4 \AA$ for $\mathrm{CH}_{3}$, and $1.44 \AA$ for $\mathrm{H}$ atoms of the hydroxyl groups. Within this approach, both the electrostatic and non-electrostatic components of the solvation energy were taken into account.

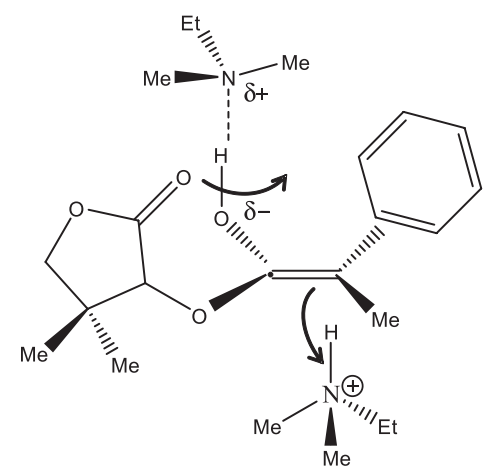

Scheme 3. Assisted (left) intramolecular (as proposed in reference 6) and (right) intermolecular proton transfers. 
In order to proceed, we will present the results for the calculations for two possible mechanisms. The first one is very simple and the second one is more complete. Although the second one is largely favored in the comparison to available experimental data, the simplicity of the first one allows us to put in evidence important non-obvious information about the system. Thus, the two mechanisms investigated are very different, and the main distinction between them is related to the origin of the proton that is transferred. In the mechanism (A), the proton comes from an intramolecular donation of the cyclic enol $\mathbf{7}$ (II) formed, as pictured in Scheme 4.

A second possibility is investigated in mechanism (B) and concerns on a two-step intermolecular proton transfer. In the first step, 7(II) has its proton abstracted by the amine present in the medium, producing the enolate intermediate $\mathbf{8}$ (Scheme 5). Another proton is donated to intermediate $\mathbf{8}$ from the ammonium salt (formed in situ when the ketene is produced). The order of such events should not be inverted, because the amine in the first step helps to block the access to one side of the double bond. With this assumption, such proposition explains the enantiomeric excess experimentally observed. Additionally, this mechanism precludes the necessity of rotation proposed by Cannizzaro et al.${ }^{6,9}$ as a justification for any enantiomeric preference.

Summarizing, the conversion of the enol intermediate 7(II) into pantolactonyl esters $(S, R)-\mathbf{6}$ and $(R, R)-\mathbf{6}$ could in principle happen through two routes: the intra (A) or intermolecular (B) protonation of the $\mathrm{C}=\mathrm{C}$ bond.
It is necessary to establish parameters to evaluate the proposed mechanisms. A sensible choice implies on the consideration of the theoretical value of the diastereoisomeric excess. The mechanism that yields a value of diastereoisomeric excess closer to the experimental data, should be the most reasonable. The theoretical evaluation of the diastereoisomeric excess can be performed through a calculation of the transition states free energies of the competing reaction pathways. The fundamental equation of the transition state theory relates the reaction rate constant $k(\mathrm{~T})$ exclusively to the free energy of the transition state: ${ }^{28}$

$k=\left(k_{B} T / h\right) \exp \left(-\Delta G^{ \pm}{ }_{r e l} / R T\right)$

The symbol $\Delta G^{*}{ }_{r e l}$ indicates the free energy difference between the transition state and an arbitrarily chosen reference energy (in this case the enol 7(II) intermediate). The constants $h$ and $k_{B}$ are Planck and Boltzmann constants, respectively. Therefore, the ratios between the configuration of the products $(R, R)-\mathbf{6}$ and $(S, R)-\mathbf{6}$ is given by

$[R, R] /[S, R]=\exp \left(-\Delta G^{\ddagger}{ }_{R} / R T\right) / \exp \left(-\Delta G_{S}^{ \pm} / R T\right)$

The percentual diastereoisomeric excesses can be calculated as follows:

$e e=\frac{[R R]-[S R]}{[R R]+[S R]} \times 100=\frac{\frac{[R R]}{[S R]}-1}{\frac{[R R]}{[S R]}+1} \times 100$<smiles></smiles>

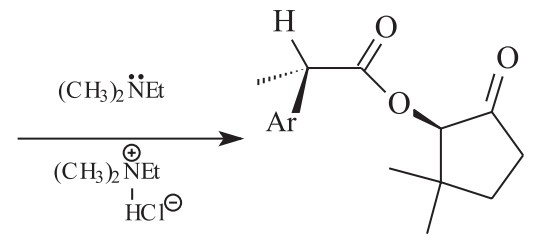

Scheme 4. Mechanism (A): direct intramolecular proton transfer.

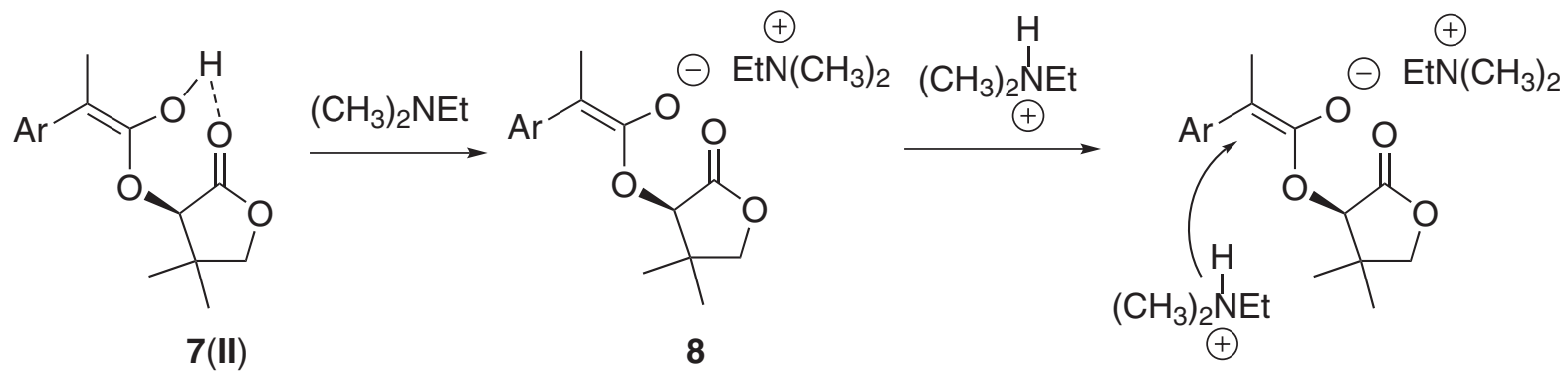

Scheme 5. Mechanism (B): an intermolecular proton transfer, catalyzed by an amine molecule, and an ammonium salt in two successive steps. 


\section{Results and Discussion}

In both mechanisms investigated, the starting structure is the enol intermediate 7(II). It can form a seven-member ring through a hydrogen bond interaction. Due to this, such structure was investigated in calculations that scanned all possible values for the dihedral angle $\phi(\phi=\mathrm{C} 1-\mathrm{C} 2-\mathrm{O}-\mathrm{C} 3)$, which defines the relative orientation of the lactone moiety in relation to the ketene moiety, through a rotation around the C3-O bond, as shown in Figure 1.

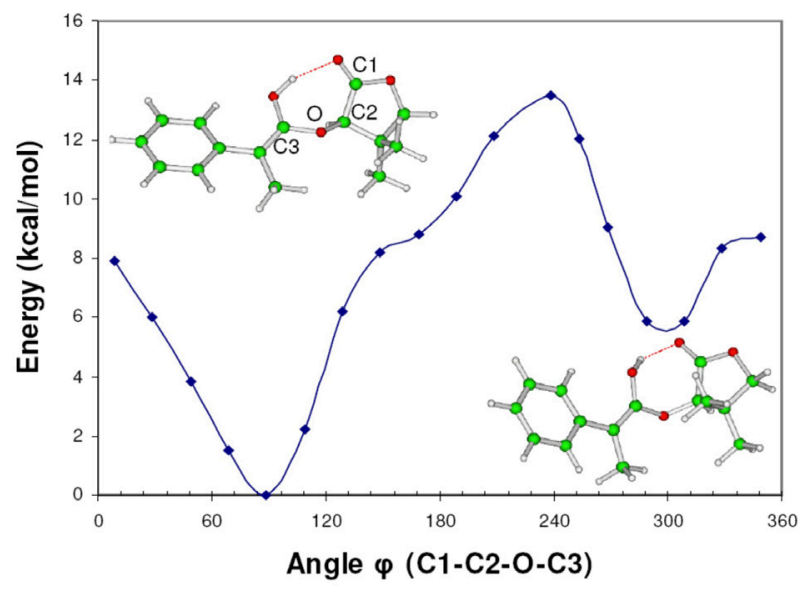

Figure 1. Potential curve calculated for the angle $(\phi=\mathrm{C} 1-\mathrm{C} 2-\mathrm{O}-\mathrm{C} 3)$ of the enol identified in Scheme 2 as 7(II).

From Figure 1 it can be seen that the potential energy curve for this enol intermediate 7(II) shows as global minimum a seven-member ring with an hydrogen bond intramolecular interaction. This strong interaction is maximized in this conformation, because the oxygen atom of the hydroxyl group is parallel to the carbonyl.
The structure corresponding to the global minimum was completely optimized, and the geometrical parameters remained basically unchanged. The most relevant geometrical parameters of this structure are shown in Figure 2.

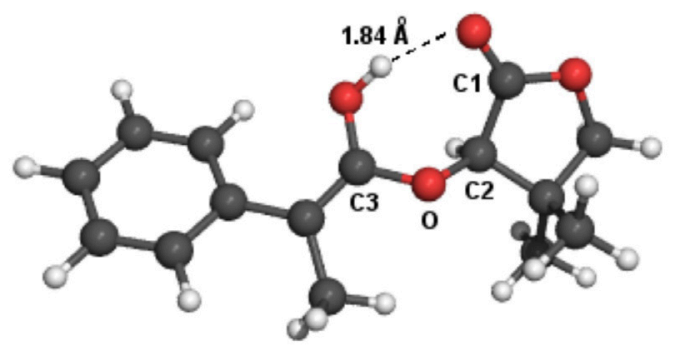

Figure 2. The enol intermediate 7(II) geometry.

The intramolecular mechanism (A)

In the intramolecular protonation mechanism the hydroxyl group revolves around the $\mathrm{C} 3-\mathrm{OH}$ bond to bring the hydrogen atom closer to the bond $\mathrm{C} 3=\mathrm{C} 4$. Then, the proton transfer to carbon atom $\mathrm{C} 4$ occurs. Such motion clearly implies the breaking of the seven-member ring structure. The critical energies (electronic energy added to zero point correction energy) and transition states structures for the conversion of this intermediate into the $(S, R)-\mathbf{6}$ and $(R, R)-\mathbf{6}$ are shown in Figure 3 .

It is clearly seen that this intramolecular process demands a very high amount of energy input. We consider it to be a reasonable result due to all the necessary structural changes discussed above. The transition state structure for $(S, R)-6$ is less stable than that one for $(R, R)-6$ because in the first mechanism an additional rotation of the $\left(\mathrm{C}_{6} \mathrm{H}_{5}\right)$

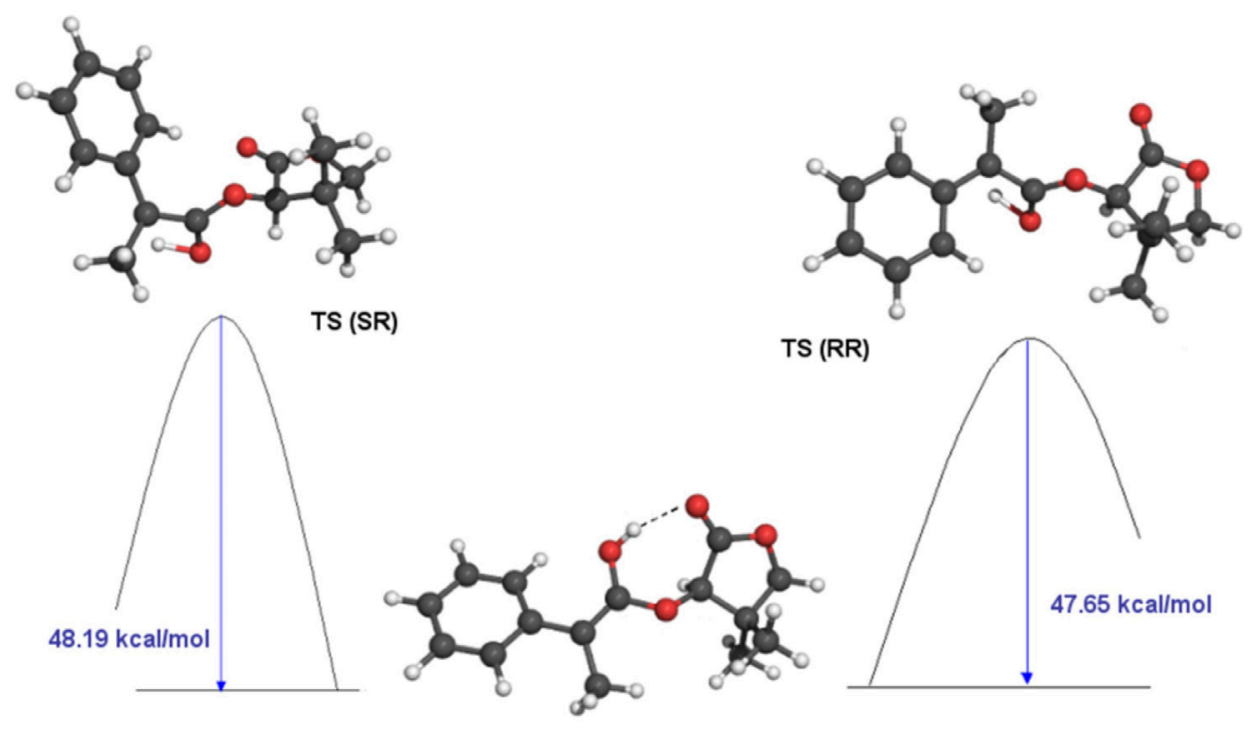

Figure 3. Reactions barriers for the proton transfer in the intramolecular mechanism (A). 
$\left(\mathrm{CH}_{3}\right)-\mathrm{C} 4$ fragment around the $\mathrm{C} 3-\mathrm{C} 4$ bond is necessary to furnish the desired stereochemistry.

After inserting the results obtained into equations 2 and 3, we found out that the difference between the barriers height was only $0.54 \mathrm{kcal} / \mathrm{mol}$. Thus, for this intramolecular mechanism we obtained a value of 57:43 for the diastereoisomeric $(S, R)-\mathbf{6} /(R, R)-6$ excess, which is too far from the experimental data $(99: 1) .^{5}$ In other words, as the height of the barriers is very similar, there is no diastereoisomeric excess associated with mechanism (A), practically. It also can be argued that the proton is too small to be responsible for observed enantioselectivity. The fact that the calculated barriers are somewhat high and very similar in magnitude provides favorable conditions for proton tunneling. ${ }^{29}$ Even in this case there would be no significant modification in the calculated diastereoisomeric excess. It is important to recall that in mechanism (A) it was considered that the amine molecule played no role in the intramolecular proton transfer. This is different from what was proposed by Canizzaro and Houk. ${ }^{6}$ However, in both propositions, the transferred proton comes from the chiral alcohol, which in the present case is the $(R)$-pantolactone. It is essential to bear in mind that the reaction studied in this paper includes a proton transfer step in solution that only take place in the presence of a proton carrier (the amine molecule) in a definite concentration. Hence, a meaningful mechanism must consider the acid-base equilibria with the proton carrier, knowing that in solution both protonated and deprotonated forms are present and are probably also active in the mechanism. To accomplish that, we move to the description of the intermolecular mechanism (B).

\section{The intermolecular mechanism (B)}

In this mechanism we have considered an intermolecular proton transfer assisted by the amine and the ammonium salt, both acting as catalysts of the reaction. It is known that for some reactions in condensed phase, like the glucose mutarotation in aqueous solution, ${ }^{30}$ the solvent-assisted pathway is much more favored than the intramolecular pathway. Taking into account this possibility, and once the ammonium salt can act as another proton donator besides the chiral alcohol, the following steps were investigated.

\section{Diastereoisomer $(S, R)-6$}

Firstly, the interaction of the enol intermediate 7(II) with dimethylethylamine molecule was studied. When this tertiary amine gets closer to 7(II) over the seven-member ring, there is an acid-base interaction between the hydroxyl group and the nitrogen atom that disrupts the hydrogen bond in the ring and forms an acid-base complex, which structure is shown in Figure $4 \mathrm{a}$. The distance $\mathrm{N} 1 \cdots \mathrm{H} 3$ is $1.74 \AA$.

\section{Enolate formation}

Dimethylethylamine is basic enough to remove the proton $\mathrm{H} 3$ through an acid-base reaction. Figure $4 \mathrm{~b}$ shows the transition state of this reaction. The $\mathrm{O} 3-\mathrm{H} 3$ bond distance is enlarged from $1.01 \AA$ to $1.22 \AA$, and N1 $\cdots \mathrm{H} 3$ distance is shortened from $1.74 \AA$ to $1.28 \AA$, both in the transition states after dimethylethylamine molecule approaches (Table 1). The reaction barrier for this step is only $0.95 \mathrm{kcal} / \mathrm{mol}$. In practice this result indicates an absence of barrier. The imaginary vibrational frequency of this transition state is $824.5 \mathrm{i} \mathrm{cm}^{-1}$, and the main components of these frequency modes are the O3-H3 and N1-H3 stretchings, as expected for a proton-migration reaction.

As can be observed from Table 1, the C3-O3 bond starts to have a double bond character, changing its length from $1.34 \AA$ (a) to $1.28 \AA$ (c), while the C3-C4 is slightly enlarged.

Table 1. Geometrical parameters (in $\AA$ ) for the structures of the reaction path of Figure $4(S, R)-6$

\begin{tabular}{lcccccc}
\hline & \multicolumn{7}{c}{ Structure } \\
\cline { 2 - 7 } Distances & (a) & (b) & (c) & (d) & (e) & (f) \\
\hline $\mathrm{O}-\mathrm{C} 3$ & 1.38 & 1.41 & 1.42 & 1.43 & 1.42 & 1.40 \\
$\mathrm{C} 3-\mathrm{O} 3$ & 1.34 & 1.31 & 1.28 & 1.23 & 1.23 & 1.23 \\
$\mathrm{O} 3-\mathrm{H} 3$ & 1.01 & 1.22 & 1.47 & - & - & - \\
$\mathrm{N} 1-\mathrm{H} 3$ & 1.74 & 1.28 & 1.11 & - & - & - \\
$\mathrm{C} 3-\mathrm{C} 4$ & 1.35 & 1.36 & 1.37 & 1.42 & 1.44 & 1.52 \\
$\mathrm{~N} 2-\mathrm{H} 2$ & - & - & - & 1.10 & 1.18 & 2.59 \\
$\mathrm{C} 4-\mathrm{H} 2$ & - & - & - & 1.85 & 1.66 & 1.10 \\
\hline
\end{tabular}

\section{(S, R)-6 formation}

After the enolate (structure (c)) formation, the dimethylethylammonium remains in the $\mathrm{O} 1$ carbonyl oxygen vicinities (not shown in Figure 4), and a second dimethylethylammonium molecule (generated in situ when the ketene is formed), attacks the $\mathrm{C} 4$ carbon atom by the opposite side, defining the stereochemistry of the final product. The structure of the reactive complex between enolate and this second dimethylethylammonium is shown in Figure $4 \mathrm{~d}$. The optimized structure of the transition state for the $\mathrm{C} 3=\mathrm{C} 4$ bond protonation is shown in Figure 4e. This transition state is a $(S, R)-6$ diastereoisomer. The N2-H2 bond is enlarged from $1.10 \AA$ to $1.18 \AA$, meanwhile the distance $\mathrm{C} 4 \cdots \mathrm{H} 2$ is shortened from $1.85 \AA$ to $1.66 \AA$. The reaction barrier of this step is $0.20 \mathrm{kcal} / \mathrm{mol}$. The imaginary 


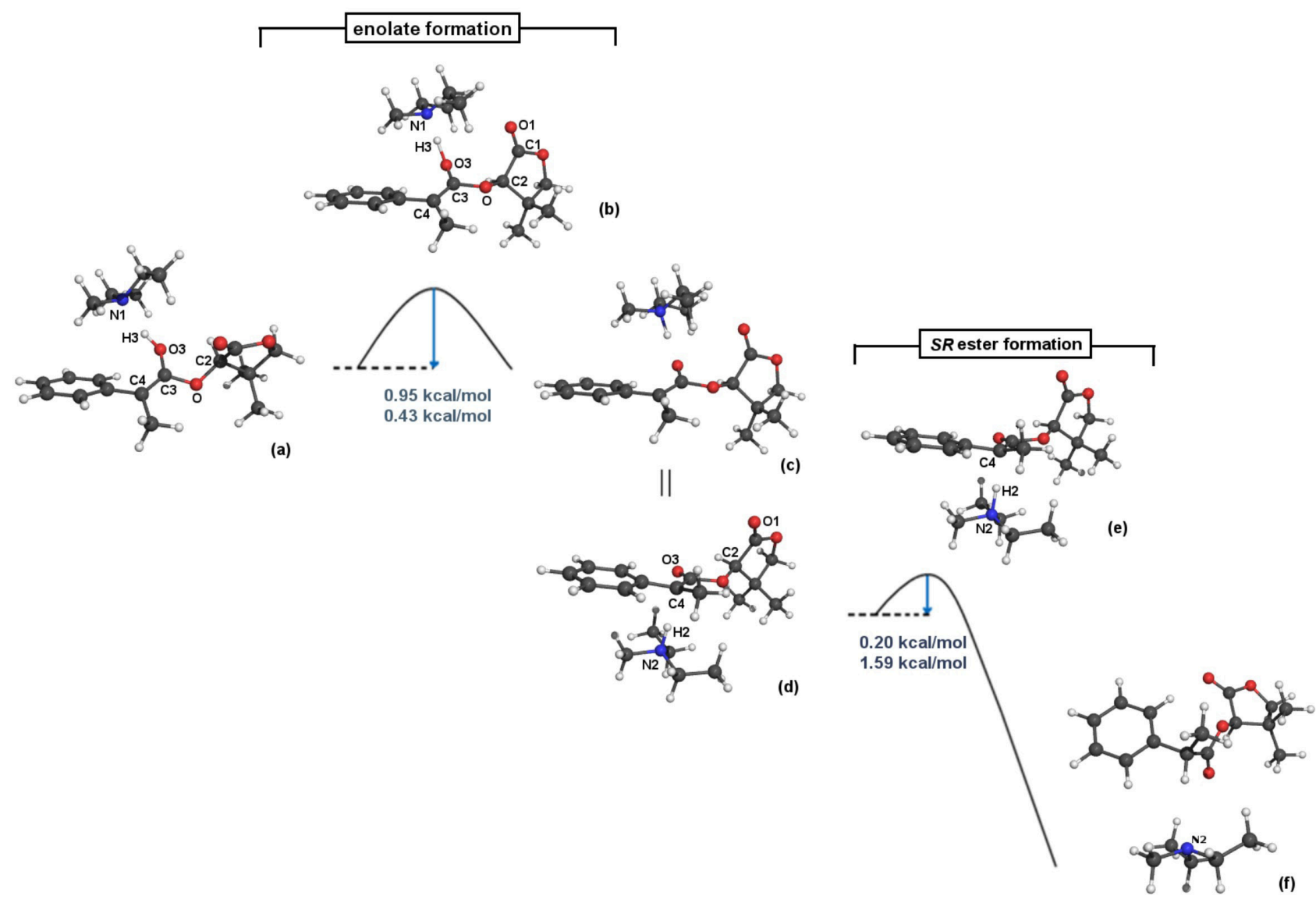

Figure 4. Optimized structures for diastereoisomer $(S, R)$ : (a) the interaction complex between the enol intermediate and dimethylethylamine, (b) transition state and (c) enolate; (d) interaction complex between the enolate and the ammonium ion, (e) transition state and (f) $(S, R)$ final product. Structures (a), (c), (d) and (f) were obtained from an IRC calculation. Relative energies values are at B3LYP and MP2 levels, respectively.

vibrational frequency of this transition state is $489.1 \mathrm{i} \mathrm{cm} \mathrm{cm}^{-1}$, and the main components of the modes of this frequency are the N2-H2 and $\mathrm{C} 4-\mathrm{H} 2$ stretchings.

As can be seen from Table 1, the C3-C4 bond character changes from a double to a single bond, while its length enlarges from $1.42 \AA$ (a) to $1.52 \AA$ (c). C4-H2 bond length is shortened simultaneously. The optimized structures of $(S, R)-6$ and the dimethylethylamine molecule (the reaction products) are shown in Figure $4 \mathrm{f}$. The distance $\mathrm{N} 2 \cdots \mathrm{H} 2$ is enlarged to $2.59 \AA$. The products are more stable than the reactive complex by $29.3 \mathrm{kcal} / \mathrm{mol}$, resulting in an essentially irreversible reaction.

Comparing the height of the barriers for enolate formation and $(S, R)$ ester formation (Figure 4$)$, it can be said that the enolate generation is the rate determining step, being the one used in the calculation of the rate coefficient.

In summary, according to this mechanism, we can picture the diastereoselective addition of $(R)$-pantolactone to isopropylphenylketene as occurring via acid-base reactions in two intermolecular proton transfer steps. It is important to mention that the proton transferred to the planar carbon comes from the dimethylethylammonium molecule which approaches from the opposite side to carbonyl group of $(R)$-pantolactone, which by its turn, comes from the alkyl halide used to generate the ketene (and not from the pantolactone).

\section{Diaestereoisomer $(R, R)-6$}

In this case, the interaction of the enol intermediate 7(II) with the dimethylethylamine molecule approaching by the opposite side was studied. When this tertiary amine gets closer to 7(II) over the seven-member ring, again there is an acid-base interaction between the hydroxyl group and the nitrogen atom that disrupts the hydrogen bond in the ring, twisting the hydroxyl to the same side of amine, and forming an acid-base complex, which structure is shown in Figure 5a. The distance N2 $\cdots \mathrm{H} 3$ is of $1.72 \AA$.

\section{Enolate formation}

As discussed previously, dimethylethylamine is able to remove the proton $\mathrm{H} 3$ through an acid-base reaction. Figure $5 b$ shows the transition state for this reaction. The 
O3-H3 bond distance is enlarged from $1.01 \AA$ to $1.32 \AA$, and $\mathrm{N} 1 \cdots \mathrm{H} 3$ distance is shortened from $1.74 \AA$ to $1.18 \AA$, both in the transition states after dimethylethylamine approach. Comparing such values to those of Table 1 for the $(S, R)$ enolate formation, it is possible to see that the N1-O3 distance is the same, being the H3 proton in the $(S, R)$ transition structure closer to the $\mathrm{O} 3$ than in the $(R, R)$ enolate. Table 2 and Figure 5 show all optimized structures involved in this reaction pathway for the $(R, R)$ product. The reaction barrier is $3.97 \mathrm{kcal} / \mathrm{mol}$. The imaginary vibrational frequency is $254.4 \mathrm{i} \mathrm{cm}^{-1}$, corresponding to the $\mathrm{O} 3-\mathrm{H} 3$ and N1-H3 bond stretchings.

In the elementary reaction responsible for the $(S, R)-6$ (Figure $4 \mathrm{e}$ ), it can be seen that the dimethylethylamine molecule interacts simultaneously with the hydroxyl group and with the carbonyl group of the pantolactone, getting more stuck than in the $(R, R)-6$ formation. In the former case, the conversion from the reactive complex to the transition state demands an energy cost almost exclusively to cleave the $\mathrm{O} 3-\mathrm{H} 3$ bond, followed by the transfer of the $\mathrm{H} 3$ proton to the $\mathrm{N} 1$ nitrogen atom.
In the case of the $(R, R)-6$ formation, however, the dimethylethylamine molecule interacts only with the hydroxyl group (Figure 5c), since the carbonyl of the pantolactone is in an opposite side. In this case the dimethylethylamine molecule has much more mobility and, consequently, more energy is necessary for the conversion of the reactive complex to the transition state. It is so because beyond the energy cost to activate the $\mathrm{O} 3-\mathrm{H} 3$ bond cleavage, it is necessary to do an extra effort for the orientation and transportation of the dimethylethylamine to the hydroxyl group.

The difference of $3.02 \mathrm{kcal} / \mathrm{mol}$ between the height of the barriers of this reaction and that schematically summarized in Figure 4 can be attributed to the very specific way the dimethylethylamine molecule interacts with the enol intermediate.

\section{(R, R)-6 formation}

It is assumed again that after the formation of enolate $\mathbf{8}$ the dimethylethylammonium remains close to it, due to

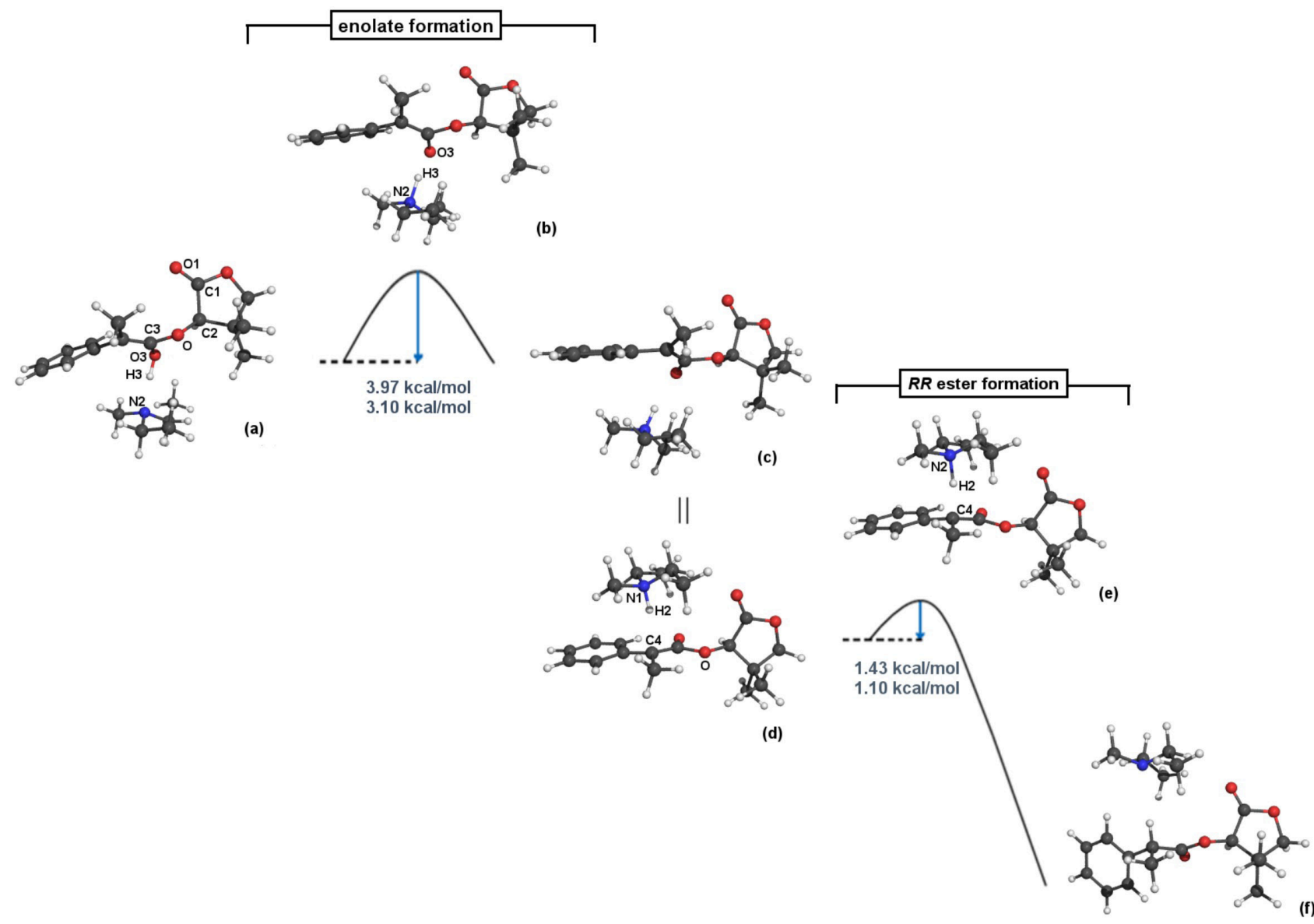

Figure 5. Optimized structures for diastereoisomer $(R, R)$ : (a) the interaction complex between the enol intermediate and dimethylethylamine, (b) transition state and (c) enolate; (d) interaction complex between the enolate and the ammonium ion, (e) transition state and (f) $(R, R)$ final product. Structures (a), (c), (d) and (f) were obtained from an IRC calculation. Relative energies values are at B3LYP and MP2 levels, respectively. 
Table 2. Geometrical parameters (in $\AA$ ) for the structures of the reaction path of Figure $5(R, R)-6$

\begin{tabular}{lcccccc}
\hline & \multicolumn{7}{c}{ Structure } \\
\cline { 2 - 7 } Distances & (a) & (b) & (c) & (d) & (e) & (f) \\
\hline O-C3 & 1.39 & 1.41 & 1.42 & 1.43 & 1.41 & 1.37 \\
C3-O3 & 1.35 & 1.30 & 1.26 & 1.23 & 1.22 & 1.20 \\
O3-H3 & 1.01 & 1.32 & 1.93 & - & - & - \\
N2-H3 & 1.72 & 1.18 & 1.02 & - & - & - \\
C3-C4 & 1.35 & 1.36 & 1.38 & 1.41 & 1.44 & 1.52 \\
N2-H2 & - & - & - & 1.01 & 1.24 & 2.65 \\
C4-H2 & - & - & - & 1.95 & 1.57 & 1.01 \\
\hline
\end{tabular}

electrostatic interactions. That opens the possibility for $\mathbf{8}$ to be attacked by a second dimethylethylammonium molecule (generated in situ), in the opposite side of that the first proton was transferred to. The structure of the reactive complex between $\mathbf{8}$ and this second dimethylethylammonium ion is shown in Figure $5 \mathrm{~d}$. The optimized structure of the transition state for the $\mathrm{C} 3=\mathrm{C} 4$ bond protonation is shown in Figure 5e. This transition state is an $(R, R)$ diastereoisomer. The reaction barrier of this step is $1.43 \mathrm{kcal} / \mathrm{mol}$. The imaginary vibrational frequency of this transition state is $1007.5 \mathrm{i} \mathrm{cm}^{-1}$, corresponding to N2$\mathrm{H} 2$ and $\mathrm{C} 4-\mathrm{H} 2$ stretchings.

It should be mentioned that in the $(R, R)-6$ formation, following this mechanism, the dimethylethylammonium that donates the proton to the planar carbon gets closer to the nucleophilic center located in the vicinities of the carbonyl of the pantolactone, and this could retard this attack.

After inserting our data into equations 2 and 3, the obtained difference between the barriers heights for the enolate formation (the rate determining step in this bimolecular mechanism) is $3.02 \mathrm{kcal} / \mathrm{mol}$. Hence, for this bimolecular mechanism we obtain a diastereoisomeric excess $S R / R R$ value above 99:1, which is in very good agreement with experimental results. ${ }^{5}$

In Figures 4 and 5 we also compare the B3LYP and MP2 results for reaction barriers. Both methods agree on the fact that the barriers for the formation of the $(S, R)$ isomer are lower than the barriers for the formation of the $(R, R)$ one. Even so, there are some noticeable differences between the two descriptions. The main physical advantage of an MP2 calculation in relation to a DFT/B3LYP one is that in the former the dispersion effects among the electrons are included in an approximate way. Thus, differences in the reaction's barriers may be associated with differential dispersion effects ascribed to each calculation type. However, we believe that these differences are of no practical consequence for the mechanism proposed in this paper. They are all essentially absorbed by the ZPE corrections causing no alteration whatsoever in our proposition.

\section{The solvation effects}

Considering the small values found for the reaction barriers, the differential solvation effects should necessarily be taken into account. Beside that, the compounds could react under different ways in solution, and this possibility does not give place to any kind of generalizations about the solvent effects in the reactions studied.

All the structures obtained in the previous calculations of the intermolecular mechanism have been re-optimized under the solvation effect. These new results show that the geometrical parameters of the structures of the intermolecular mechanism are almost identical to those listed in Tables 1-4. The most significant changes occur in the values of the reaction barriers, as can be seen from data reported in Table 3.

Table 3. Solvent effects on the reaction paths of the intermolecular mechanism (B) studied. Relative energies are in $\mathrm{kcal} / \mathrm{mol}$, and the MP2 values between parentheses follow DFT values

\begin{tabular}{lcccc}
\hline \multirow{2}{*}{ Formation step } & \multicolumn{4}{c}{ Without ZPE } \\
\cline { 2 - 5 } Enolate & $(S, R)$ & $(R, R)$ & $(S, R)$ & $(R, R)$ \\
\cline { 2 - 5 } Ester & 0.95 & 3.97 & 0.41 & 1.23 \\
& $(0.43)$ & $(3.10)$ & $(0.46)$ & $(1.33)$ \\
& 0.20 & 1.43 & 0.64 & 2.94 \\
& $(1.59)$ & $(1.10)$ & $(-1.36)$ & $(0.32)$ \\
\hline \multirow{5}{*}{ Enolate } & 0.46 & 2.74 & -0.84 & 0.28 \\
& $(-0.73)$ & $(2.21)$ & $(-0.70)$ & $(0.41)$ \\
Ester & -1.75 & 0.35 & -0.51 & 2.20 \\
& $(0.35)$ & $(0.12)$ & $(-1.45)$ & $(-0.85)$ \\
\hline
\end{tabular}

From the B3LYP and MP2 descriptions, the solvent decreases the reaction barriers of the enolate formation step in both $(S, R)$ and $(R, R)$ diastereoisomer routes. However, in the chiral ester formation step, the height of the barriers is larger in both routes if B3LYP energy values are considered, but decrease in THF if MP2 results are considered. Then, the solvation effects seem to be determinant not only to define the enantiomeric excess, but also to identify the rate-determining step in the reaction. In the gas-phase the rate-determining step is the enolate formation, but if the solvent effects are introduced in the calculations, it is no longer possible to assure this. The inclusion of ZPE corrections does not alter the prediction of the observed 
stereoselectivity. However, the resultant negative barriers preclude us from the calculation of the enantiomeric excess. The kinetics associated with reactions in multicomponent solutions is very complex. The full role of the charged species is not entirely clear. Hence, one should not expect the present kind of study, and also the other ones present in the literature, to provide the ultimate dynamic picture of all processes relevant for the outcome of the reaction. What should be expected is a proposal that does not contradicts existent data, yielding at the same time useful insights on the possible mechanism of the process in question. The very good agreement between the calculated diastereoisomeric excess (without ZPE corrections) and the experimental value gives us confidence that we are in the right track.

\section{Conclusions}

In this work the synthetic route leading to the 1-4 asymmetric induction in the preparation of 2-phenylpropionate pantolactonyl ester was theoretically investigated. It was experimentally observed that diastereoisomeric ratio $(S, R)-\mathbf{6} /(R, R)-\mathbf{6}$ is about 99:1. ${ }^{5,14}$

Two mechanisms have been considered to study the stereoselectivity: one assumes the intramolecular proton transfer, and the other assumes the intermolecular proton transfer through the assistance of dimethylethylamine and dimethylethylammonium chloride. A very stable enol intermediate bearing a seven-member ring, generated through the addition of the ketene and $(R)$-pantolactone was considered as the starting reactant in both cases. The calculations have been carried out at DFT/B3LYP and MP2 levels with the 6-311G(d,p) basis set.

The results obtained have shown that the intramolecular mechanism is not able to account for different reaction barriers that would be consistent with the experimentally observed diastereoisomeric excess. Due to the small size of the proton, it cannot be held responsible for the observed enantioselectivity since in practice the two possible available faces for the proton transfer cannot be differentially blocked. Hence this channel was discarded as a reaction path.

The intermolecular mechanism considers the active participation of the dimethylethylamine. Firstly, a neutral dimethylethylamine molecule interacts with the sevenmembered ring of the enol intermediate 7(II), and a specific acid-base interaction is established between the hydroxyl group of enol and the nitrogen atom of dimethylethylamine. The neutral dimethylethylamine is able to remove the proton to generate an enolate ion $\mathbf{8}$ and a dimethylethylammonium ion. In the sequence, another dimethylethylammonium ion already available in the reaction medium, is considered to donate its proton to the $\mathrm{C}=\mathrm{C}$ double bond to give the desired products, the $(S, R)-\mathbf{6}$ and $(R, R)-\mathbf{6}$. In the mechanism here proposed the transferred proton comes from the alkyl halide used to generate the ketene in situ, since (at least in the beginning of the reaction) the ammonium salt is obtained when hydrochloric acid is formed. According to this mechanism, the amount of amine present in the reaction medium is a key point for this reaction to work, as confirmed by experiments. ${ }^{14}$ There must be enough amine to promote the enolate formation (first and determinant step of mechanism B) but not great excess, since it would dilute the ammonium salt that attacks the planar carbon (second step of mechanism B). Mechanism B is also able to explain the role of a chiral hydroxyl, $\alpha$ to a carbonyl group, a characteristic needed for a good chirality inductor. Our main conclusion is that the specific side from which the neutral and protonated dimethylethylamine approaches to the enol intermediate defines the stereochemical outcome. The diastereoisomeric ratio is determined by the relative values of the reaction barriers heights of the protonation step of the $\mathrm{C}=\mathrm{C}$ double bond. The theoretically calculated value for the diastereoisomeric ratio agrees with the experimental result thereby confirming the soundness of the proposed mechanism.

Differently from Cannizzaro and Houk's mechanism where the stereoselectivity is determined by the addition step that generates an enol intermediate unable to rotate due to a high energy barrier, we suggest in mechanism (B) that the stereoselectivity is defined during the tautomerization, by the side that the ammonium ion is approached to the enolate. The use of polar solvents, that could solvate better the ammonium salt, would certainly decrease the diastereoselectivity of the Merck process, as in fact was observed experimentally. This proposition also explains the kinetic isotopic effect observed for deuterated alcohols ${ }^{31}$ (in this case the pantolactone), once its proton is withdrawn by the neutral amine.

The effects of solvation were also considered and their results are similar to those in gas-phase for the geometrical parameters. The noticeable changes occur in the values of the reaction barriers. The solute-solvent interaction decreases the reactions barriers of the enolate formation from 0.95 to $0.41 \mathrm{kcal} / \mathrm{mol}$ in the $(S, R)$ diastereoisomer route, and from 3.97 to $1.23 \mathrm{kcal} / \mathrm{mol}$ in the $(R, R)$ diastereoisomer route. From these results it can be inferred that the conversion from reactive complexes between amine and enol intermediate to the transition states is favored in the solvent medium.

Finally, two possible investigations can be performed to validate (B) mechanism here proposed, because in it the proton transferred to the enolate comes from the alkyl halide 
used to generate the ketene in situ. One possibility would be to deuterate the alkyl halide used and control the formation of a deuterated product. The other possibility, perhaps still more efficient, would be to work with a deuterated pantolactone. If the product and the ammonium salt in solution are found both deuterated, Cannizzaro and Houk's proposition as well as our (B) mechanism, respectively, are possible channels.

In summary, in this paper we provided an alternative mechanism for the 1-4 asymmetric induction in the preparation of 2-phenyl-propionate pantolactonyl ester. The calculated enantiomeric excess is in excellent agreement with the experimental results. The singular characteristic of the present proposal, in contrast to the ones in the literature, is the utilization of two different amine molecules as proton acceptor and donor. This brings a more realistic flavor to the proposed mechanism, since it is an experimental fact that for the reaction to take place the amine should be in a definite concentration.

\section{Acknowledgments}

Alexander M. Silva, Clarissa O. Silva and André G. H. Barbosa thank the Brazilian agency FAPERJ for financial support given to this work. Clarissa O. Silva thanks also CNPq. The authors would like to acknowledge Prof. Carlos Maurício Rabello de Sant'Anna for helpful discussions.

\section{References}

1. Weiss, R.; Mh. Chem. 1919, 40, 391.

2. Salz, U.; Ruechardt, C.; Tetrahedron Lett. 1982, 23, 4017.

3. Jähme, J.; Rüchardt, C.; Angew. Chem., Int. Ed. Engl.1981, 20, 885.

4. Bellucci, G.; Berti, G.; Bianchini, R.; Vecchiani, S.; Gazz. Chim. Ital.1988, 118, 451.

5. Larsen, R. D.; Corley, E. G.; Davis, P.; Reider, P. J.; Grabowski, E. J. J.; J. Am. Chem. Soc. 1989, 111, 7650.

6. Cannizzaro, C. E.; Houk, K. N.; J. Am. Chem. Soc. 2004, 126, 10992.

7. Calmes, M.; Daunis, J.; Jacquier, R.; Natt, F.; Tetrahedron 1994, 50,6875 .

8. Camps, P.; Perez, F.; Soldevilla, N.; Tetrahedron: Asymmetry 1998, 9, 2065.

9. Cannizzaro, C. E.; Strassner, T.; Houk, K. N.; J. Am. Chem. Soc. 2001, 123, 2668.

10. Sonawane, H. R.; Bellur, N. S.; Ahuja, J. R.; Kulkarni, D. G.; Tetrahedron: Asymmetry 1992, 3, 163.

11. Effenberger, F.; Graef, B. W.; Osswald, S.; Tetrahedron: Asymmetry 1997, 8, 2749.

12. Cram, D. J.; Whitney, T. A.; J. Am. Chem. Soc. 1967, 89, 4651.
13. Ford, W. T.; Cram, D. J.; J. Am. Chem.Soc. 1968, 90, 2606.

14. Fontes, R. A.; MSc Dissertation, UFRRJ, Brazil 2001.

15. Kenyon, W. G.; Kaiser, E. M.; Hauser, C. R.; J. Org. Chem. 1965, 43, 2937.

16. Kenyon, W. G.; Mayer, R. B.; Hauser, C. R.; J. Org. Chem. 1963, 28, 3108.

17. Becke, A. D.; J. Chem. Phys. 1993, 98, 5648.

18. Lee, C.; Yang, W.; Parr, R. G.; Phys. Rev. B 1988, 37, 785.

19. Jaguar 7.0, Schrodinger, LLC, Portland, Oregon, 2007.

20. Frisch, M. J.; Trucks, G. W.; Schlegel, H. B.; Scuseria, G. E.; Robb, M. A.; Cheeseman, J. R.; Montgomery, Jr., J. A.; Vreven, T.; Kudin, K. N.; Burant, J. C.; Millam, J. M.; Iyengar, S. S.; Tomasi, J.; Barone, V.; Mennucci, B.; Cossi, M.; Scalmani, G.; Rega, N.; Petersson, G. A.; Nakatsuji, H.; Hada, M.; Ehara, M.; Toyota, K.; Fukuda, R.; Hasegawa, J.; Ishida, M.; Nakajima, T.; Honda, Y.; Kitao, O.; Nakai, H.; Klene, M.; Li, X.; Knox, J. E.; Hratchian, H. P.; Cross, J. B.; Bakken, V.; Adamo, C.; Jaramillo, J.; Gomperts, R.; Stratmann, R. E.; Yazyev, O.; Austin, A. J.; Cammi, R.; Pomelli, C.; Ochterski, J. W.; Ayala, P. Y.; Morokuma, K.; Voth, G. A.; Salvador, P.; Dannenberg, J. J.; Zakrzewski, V. G.; Dapprich, S.; Daniels, A. D.; Strain, M. C.; Farkas, O.; Malick, D. K.; Rabuck, A. D.; Raghavachari, K.; Foresman, J. B.; Ortiz, J. V.; Cui, Q.; Baboul, A. G.; Clifford, S.; Cioslowski, J.; Stefanov, B. B.; Liu, G.; Liashenko, A.; Piskorz, P.; Komaromi, I.; Martin, R. L.; Fox, D. J.; Keith, T.; Al-Laham, M. A.; Peng, C. Y.; Nanayakkara, A.; Challacombe, M.; Gill, P. M. W.; Johnson, B.; Chen, W.; Wong, M. W.; Gonzalez, C.; Pople, J. A,; Gaussian 03, Revision C.02, Gaussian, Inc., Pittsburgh, Wallingford, CT 2004.

21. Gonzales, C.; Schlegel, H. B.; J. Chem. Phys. 1989, 90, 2154.

22. Gonzales, C.; Schlegel, H. B.; J. Phys. Chem. 1990, 94, 5523.

23. Zhao, Y.; Truhlar, D. G.; J. Chem. Phys. 2006, 125, 194101; Zhao, Y.; Truhlar, D. G.; J. Phys. Chem. A 2006, 110, 13126.

24. Nielsen, A. B.; Holder, A. J.; GaussView User's Reference v.2.0, 2000.

25. Cancès, E.; Mennucci, B.; Tomasi, J.; J. Chem. Phys. 1997, 107,3032 .

26. Miertus, S.; Scrocco, E.; Tomasi, J.; Chem. Phys. 1981, 55, 117.

27. Cammi, R.; Tomasi, J.; J. Comput. Chem. 1995, 16, 1449.

28. Alagona, G.; Ghio, C.; Persico, M.; Tomasi, S.; J. Am. Chem. Soc. 2003, 125, 10027.

29. Hammes-Schiffer, S.; Acc. Chem. Res. 2001, 34, 273.

30. Silva, A. M.; da Silva, E. C.; da Silva, C. O.; Carbohydr. Res. 2006, 341, 1029.

31. Larsen, R. D.; Corley, E. G.; Davis, P.; Reider, P. J.; Grabowski, E. J.; J. Am. Chem. Soc. 1989, 111, 7650.

Submitted: August 4, 2010

Published online: January 25, 2011 